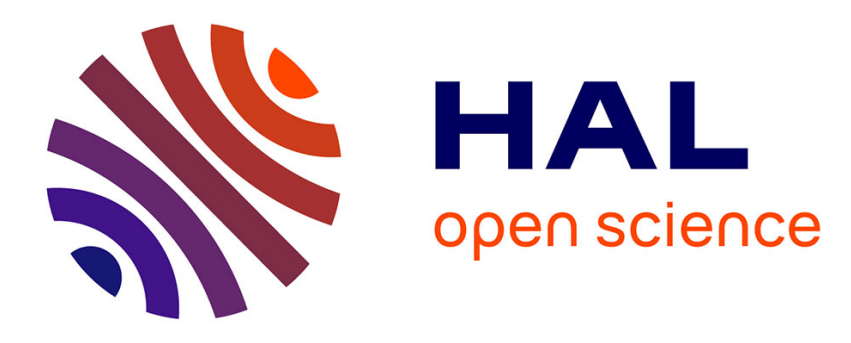

\title{
Recherches de fongicides actifs sur Stemphylium floridanum Hannon et Weber, parasite de Solanum gilo Raddli
}

Alain Clerivet

\section{- To cite this version:}

Alain Clerivet. Recherches de fongicides actifs sur Stemphylium floridanum Hannon et Weber, parasite de Solanum gilo Raddli. Agronomie, 1987, 7 (3), pp.163-166. hal-00884980

\section{HAL Id: hal-00884980 \\ https://hal.science/hal-00884980}

Submitted on 1 Jan 1987

HAL is a multi-disciplinary open access archive for the deposit and dissemination of scientific research documents, whether they are published or not. The documents may come from teaching and research institutions in France or abroad, or from public or private research centers.
L'archive ouverte pluridisciplinaire HAL, est destinée au dépôt et à la diffusion de documents scientifiques de niveau recherche, publiés ou non, émanant des établissements d'enseignement et de recherche français ou étrangers, des laboratoires publics ou privés. 


\title{
Recherches de fongicides actifs sur Stemphylium floridanum Hannon et Weber, parasite de Sola- num gilo Raddli
}

\author{
Alain CLERIVET \\ Laboratoire de Biologie végétale, Faculté des Sciences, Université Hassan II, B.P. 5366 Maârif, Casablanca,
} Maroc

\begin{abstract}
Le développement de Stemphylium floridanum, parasite de Solanum gilo est étudié en présence de différents fongicides. In vitro, les substances minérales n'ont aucun effet sur la croissance et la conidiogenèse du champignon tandis que parmi les composés organiques, le chlorothalonil, le captafol et le thirame ont l'action antifongique la plus marquée. In vivo, l'utilisation, à titre préventif, du captafol et du chlorothalonil montre que ces produits sont inefficaces et/ou phytotoxiques. Par contre l'action durable, performante et non phytotoxique du thirame à la concentration de $100 \mathrm{mg} / \mathrm{I}$ en fait un fongicide recommandable pour lutter préventivement contre la stemphyliose de $S$. gilo.
\end{abstract}

Mots clés additionnels : Conidiogenèse, croissance mycélienne, action préventive, thirame.

Effects of fungicides on Stemphylium floridanum Hannon and Weber, a parasite of Solanum gilo Raddli.

\begin{abstract}
The development of Stemphylium floridanum, a parasite of Solanum gilo, was studied in the presence of several antifungal substances. In vitro, mineral substances did not affect growth or conidiogenis of the fungus while, among organic compounds, chlorothalonil, captafol and thiram had the highest fungitoxicity. In vivo, captafol and chlorothalonil used as preventives were ineffective and/or phytotoxic. Thiram at $100 \mathrm{mg} / 1$ was the best product, with durable performance and no phytotoxic action. It could be recommanded for preventive treatment against $S$. floridanum on $S$. gilo.
\end{abstract}

Additional key words : Conidiogenesis, mycelial growth, preventive action, thiram.

\section{INTRODUCTION}

En Côte-d'Ivoire, la culture de Solanum gilo (Solanacées) est en pleine expansion et sa production fruitière en croissance constante, mais une maladie foliaire causée par Stemphylium floridanum présente un caractère préoccupant. En effet, cette affection parasitaire, permanente, occasionne des dégâts importants caractérisés par le dessèchement et l'abondante abscission des feuilles lors de la floraison et de la fructification (CLERIVET, 1982). De plus, les traitements antifongiques au manèbe, choisis par référence aux travaux de ROTEM (1968), SIVAN \& BARKAI-GOlaN (1976), GINDRAT et al. (1984) sur d'autres stemphylioses de diverses cultures maraîchères sont restés sans effet. La recherche d'une stratégie de la lutte active s'avère donc indispensable.

Ainsi, ce travail se propose d'étudier l'influence de quelques substances antifongiques sur la croissance et la conidiogenèse de $S$. floridanum et leur éventuelle action protectrice sur des plants de $S$. gilo infectés expérimentalement.

\section{MATÉRIEL ET MÉTHODES}

Des substances antifongiques, couramment utilisées dans la protection des cultures maraîchères en Côte-d'Ivoire ou préconisées dans la lutte contre les stemphylioses ont été testées. Ce sont des substances minérales (1) comme l'oxychlorure de cuivre (Cuprovit) ou le soufre micronisé (Microlux), des substances organiques (1) comme le manèbe, seul (Manèbe 80 ) ou associé au zinèbe (Mancozan : manèbe 60 p. 100, zinèbe 10 p. 100), au méthylthiophanate (Peltar: manèbe $50 \mathrm{p}$. 100 , méthylthiophanate $25 \mathrm{p} .100$ ), au

(1) Entre parenthèses : appellations commerciales. 
carbatène (Organil 66 : manèbe 64 p. 100, carbatène 16 p. 100); le captafol (Orthodifolatan), le thirame (Thirbane), la dichlofluanide (Euparène), le fenarimol (Rubigan 4), la triforine (Funginex), le chlorothalonil (Daconil flowable), le cymoxunil (Curzate), le mancozèbe (Dithane M45).

\section{A. Tests in vitro}

Une suspension titrée de conidies de $S$. floridanum (200 000 conidies par $\mathrm{ml}$ d'eau distillée stérile) est préparée à partir d'un thalle cultivé à $27^{\circ} \mathrm{C}$ pendant 12 jours sur V8 20 p. 100 à pH 7,2 (CLERIVET, 1985), puis introduite à raison de $1 \mathrm{ml}$ par bôte de Petri (90 $\mathrm{mm}$ de diamètre) contenant $20 \mathrm{ml}$ du même milieu gélosé en surfusion. Après homogénisation et refroidissement, quatre disques de papier filtre stérile (20 mm de diamètre) sont déposés à la surface du milieu. Deux disques sont imprégnés d'une suspension aqueuse de fongicide et servent de tests, les deux autres, d'eau distillée stérile, de témoins. Le taux de recouvrement des disques de papier et l'évaluation de la zone d'inhibition de croissance autour de ces disques sont régulièrement estimés afin de sélectionner les fongicides les plus actifs.

L'étude de l'influence des fongicides sur la croissance mycélienne et la conidiogenèse du parasite est conduite après leur addition au milieu nutritif stérile. L'ensemencement est réalisé par dépôt superficiel d'un fragment de thalle sporulé âgé de 12 jours. Le diamètre des thalles est régulièrement mesuré jusqu'à recouvrement total du milieu nutritif par le champignon; parallèlement, le niveau de sporulation est évalué après récolte des conidies et comptage à l'aide d'une cellule de Mallassez. Pour chaque condition expérimentale, 3 répétitions sont effectuées.

\section{B. Tests in vivo}

Ces tests sont réalisés sur des plants de $S$. gilo cultivés en pot, sur terre de forêt et maintenus dans les conditions naturelles d'humidité ( 90 à 95 p. 100) et de température $\left(30\right.$ à $\left.32{ }^{\circ} \mathrm{C}\right)$.

A l'âge de 8,12 et 16 semaines, les plants sont traités par pulvérisation foliaire d'une suspension aqueuse de fongicide ; $48 \mathrm{~h}$ après le traitement l'infection est réalisée selon la technique décrite par CLERIVET (1982). L'apparition des nécroses foliaires est ensuite surveillée, et les taches dénombrées. Les résultats expriment le nombre moyen de nécroses par feuille et pour 3 plants.

\section{RÉSULTATS}

\section{A. Sélection des fongicides actifs sur $S$. floridanum, in vitro (fig. 1)}

Le chlorothalonil, le captafol et le thirame ont la meilleure action antifongique. L'activité du chlorothalonil à partir de $500 \mathrm{mg} / \mathrm{l}$ est élevée et marquée par une zone d'inhibition de $2 \mathrm{~mm}$ autour du disque test. L'observation des cultures âgées de $14 \mathrm{j}$ montre les mêmes résultats.
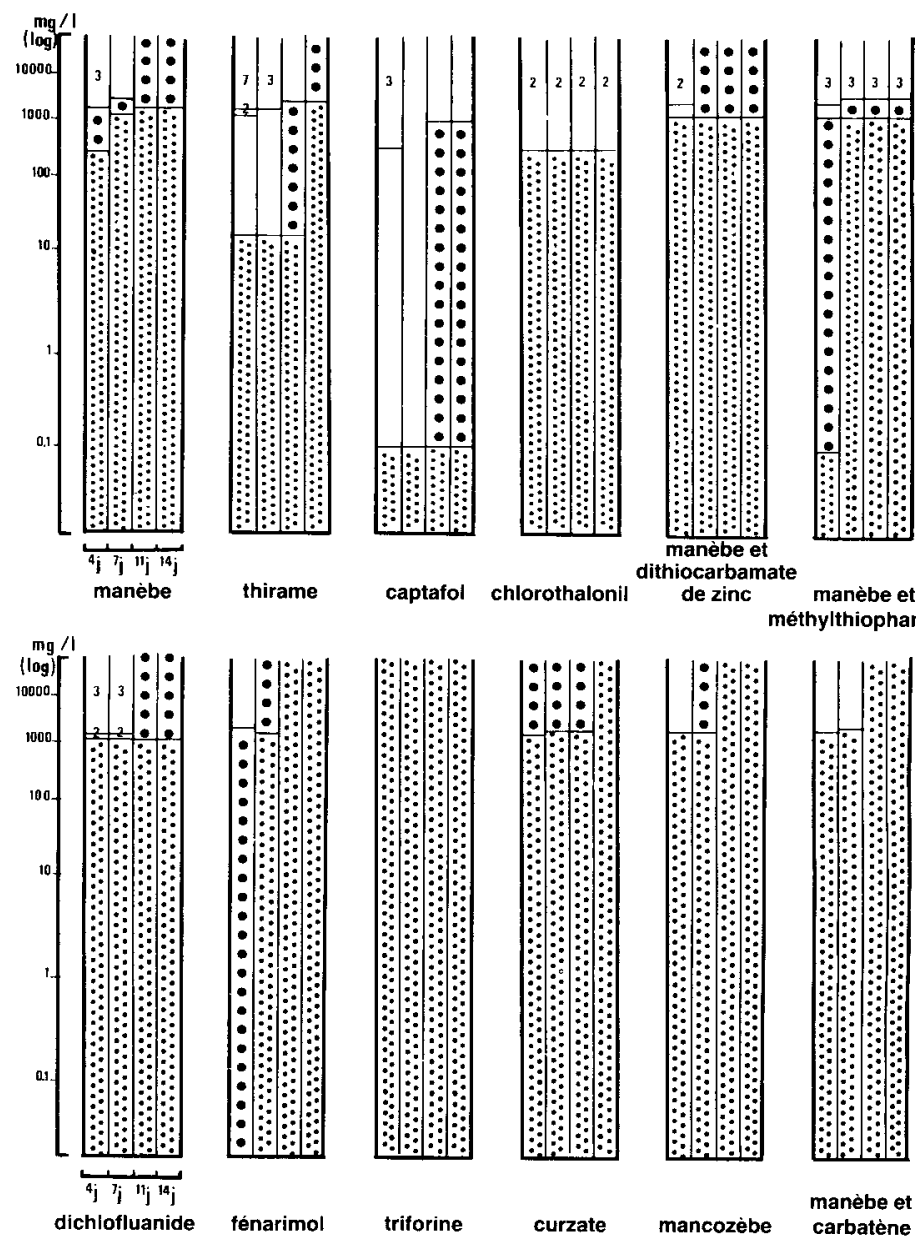

Figure 1

Action de différents fongicides sur la croissance de $\mathrm{S}$. floridanum. $\because \because=100 \mathrm{p} .100$ de recouvrement du test $; \cdots=50 \mathrm{p} .100$,

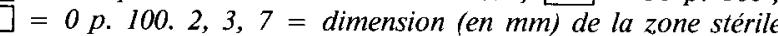
autour du test.

Action of different fungicides at different doses on growth of S. floridanum. $\because \because=$ test papers $100 \%$ overgrown; $\cdots=50 \%$; $\square=0 \% ; 2,3,7=$ size (in $\mathrm{mm}$ ) of zone of inhibition around the test paper.

Le thirame, à partir de $10 \mathrm{mg} / \mathrm{l}$ et le captafol dès $0,1 \mathrm{mg} / \mathrm{l}$ ont une action semblable à celle du chlorothalonil. Cependant, les observations effectuées au $14^{\mathrm{e}} \mathrm{j}$ de culture montrent que le champignon recouvre progressivement le disque test. Pour s'opposer à cet envahissement, les concentrations en thirame ou en captafol doivent être nettement supérieures à celles utilisées avec le chlorothalonil ( 3500 et $1500 \mathrm{mg} / \mathrm{l}$ respectivement). Les produits comme la dichlofluanide, le fénarimol et le manèbe, seul, ou associé au zinèbe ou au méthylthiophanate, doivent être employés à des doses trop élevées pour obtenir un effet antifongique durable.

Quant à la triforine, au cymoxunil et à l'association du manèbe au carbatène, leur activité est faible ou nulle.

\section{B. Influence de quelques fongicides sur la croissance mycélienne et la conidiogenèse de $S$. floridanum, in vitro}

Le captafol, le thirame, le chlorothalonil et le manèbe sont retenus. Bien qu'ayant montré un intérêt 
moindre lors des tests sélectifs, le manèbe est retenu en raison de son utilisation maraîchère contre diverses stemphylioses.

Quel que soit le produit utilisé, l'augmentation de sa concentration ralentit fortement la croissance du champignon. Pour des concentrations faibles allant jusqu'à $1 \mathrm{mg} / \mathrm{l}$ le chlorothalonil provoque le retard de croissance le plus important ; pour des doses supérieures, le captafol est le plus actif des fongicides. En présence de $200 \mathrm{mg} / 1$ de thirame ou de captafol et de $400 \mathrm{mg} / \mathrm{l}$ de manèbe, le champignon ne se développe pas. A ces concentrations, le manèbe et le captafol inhibent la germination des conidies et la croissance des hyphes mais n'ont pas d'effet létal : en effet, tous les inoculums transférés sur un milieu identique mais exempt de fongicide reprennent leur développement. Par contre, le thirame à $200 \mathrm{mg} / 1$ est létal pour 50 p. 100 des inoculums ayant subi un transfert semblable (tabl. 1).

Quel que soit le produit utilisé, des doses très faibles $(0,0001 \mathrm{mg} / \mathrm{l})$ ralentissent nettement la conidiogenèse. Ce phénomene est d'autant plus accentué que la concentration en antifongique est plus forte. La conidiogenèse est inhibée avec $200 \mathrm{mg} / \mathrm{l}$ de captafol ou de thirame et $400 \mathrm{mg} / 1$ de manèbe. Par contre, il faut $1000 \mathrm{mg} / 1 \mathrm{de}$ chlorothalonil pour obtenir cet effet (tabl. 2).

\section{Etude de l'action préventive de quelques fongi- cides (tabl. 3)}

Le chlorothalonil, le captafol, le manèbe et le thirame sont utilisés à des concentrations pour lesquelles la croissance et la conidiogenèse de $S$. floridanum sont les plus diminuées, c'est-à-dire de $10 \mathrm{mg} / 1$ à $3000 \mathrm{mg} / \mathrm{l}$. Les résultats sont semblables quel que soit l'âge des plants inoculés.

Le thirame est le fongicide le plus efficace : les feuilles de $S$. gilo traitées par une solution à $100 \mathrm{mg} / \mathrm{l}$ restent saines pendant toute la durée de l'expérience. Le manèbe, à partir de $600 \mathrm{mg} / \mathrm{l}$ retarde nettement l'apparition des symptômes de la maladie : pour observer un effet comparable à celui du thirame, la concentration doit être de $3000 \mathrm{mg} / \mathrm{l}$ mais il y a alors phytotoxicité. L'activité du captafol se situe à des concentrations provoquant des brûlures du limbe. Le chlorothalonil n'a aucune action protectrice.
TABLEAU 1

Influence de la concentration des fongicides (en $\mathrm{mg} / \mathrm{l}$ ) sur la croissance de S. floridanum.

Influence of fungicide concentration (in $\mathrm{mg} / \mathrm{l}$ ) on growth of S. floridanum.

\begin{tabular}{lllrrr}
\hline \hline & \multicolumn{5}{c}{ Croissance mycélienne (p. 100 témoin) } \\
\cline { 2 - 6 } \multicolumn{1}{c}{ Fongicide } & 90 & 75 & 50 & 25 & 0 \\
\hline Thirame & 0,1 & 1 & 10 & 100 & 200 \\
Captafol & 0,1 & 1 & 5 & 10 & 200 \\
Chlorothalonil & 0,001 & 0,01 & 1 & 100 & $>1000$ \\
Manèbe & 0,001 & 0,01 & 150 & 300 & 400 \\
\hline
\end{tabular}

TABLEAU 2

Influence de la concentration des fongicides (en $\mathrm{mg} / \mathrm{l}$ ) sur la conidiogenèse de S. floridanum.

Influence of fungicide concentration (in $\mathrm{mg} / \mathrm{l}$ ) on conidiogenesis of $\mathrm{S}$. fToridanum.

\begin{tabular}{llllrr}
\hline \multirow{2}{*}{ Fongicide } & \multicolumn{5}{c}{ Production de conidies (p. 100 témoin) } \\
\cline { 2 - 6 } & \multicolumn{1}{c}{90} & 75 & 50 & 25 & 0 \\
\hline Thirame & $<0,0001$ & 0,001 & 0,5 & 1 & 200 \\
Captafol & $<0,0001$ & 0,0001 & 1 & 5 & 200 \\
Chlorothalonil & $<0,0001$ & 0,0001 & 0,1 & 200 & 1000 \\
Manèbe & $<0,001$ & 1 & 10 & 150 & 400 \\
\hline \hline
\end{tabular}

\section{DISCUSSION ET CONCLUSIONS}

Les fongicides minéraux n'ont pas d'activité sur le développement de $S$. floridanum. Un résultat similaire est constaté avec de nombreux autres Stemphylium à l'exception de $S$. botryosum sur tomate, dont la sensibilité aux composés cupriques a été mise en évidence par ROTEM (1968)

Les fongicides de synthèse ont une activité variable sur la croissance mycélienne et la conidiogenèse, celleci étant généralement plus sensible. Dans les tests in vitro, le thirame, le chlorothalonil et le captafol sont les plus efficaces. Dans les essais in vivo, le thirame est le plus intéressant puisque son action préventive est totale et persistante, sans effets phytotoxiques à la concentration de $100 \mathrm{mg} / \mathrm{l}$. Un autre dithiocarbamate, le

TABLEAU 3

Action préventive de quelques fongicides sur $\mathrm{S}$. gilo infecté expérimentalement $10=$ pas de taches ; $1=1$ à 5 taches; $2=6$ à 10 taches; $3=11$ à $15 ; 4=16$ à $20 ; 5=$ plus de $20 .^{*}=$ phytotoxicité .

Preventive action of some fungicides on $\mathrm{S}$. gilo experimentally infected $10=$ no spots; $1=1$ to 5 spots; $2=6$ to 10 spots $3=11$ to 15 spots $; 4=16$ to 20 spots $; 5=$ more than 20 spots. ${ }^{*}=$ phytotoxicity).

\begin{tabular}{|c|c|c|c|c|c|c|c|c|c|c|c|c|c|c|}
\hline \multirow{2}{*}{$\begin{array}{l}\text { Concentration } \\
(\mathrm{mg} / \mathrm{l})\end{array}$} & \multicolumn{2}{|c|}{10} & \multicolumn{2}{|c|}{100 à 200} & \multicolumn{2}{|c|}{400} & \multicolumn{2}{|c|}{600 à 800} & \multicolumn{2}{|c|}{1000} & \multicolumn{2}{|c|}{2000} & \multicolumn{2}{|c|}{3000} \\
\hline & 8 & 15 & 8 & 15 & 8 & 15 & 8 & 15 & 8 & 15 & 8 & 15 & 8 & 15 \\
\hline Thirame & 1 & 1 & 0 & 0 & 0 & 0 & 0 & 0 & 0 & 0 & 0 & 0 & 0 & 0 \\
\hline Captafol & 5 & 5 & 5 & 5 & 5 & 5 & $4^{*}$ & $4^{*}$ & $1^{*}$ & $1^{*}$ & $0^{*}$ & $0^{*}$ & $0^{*}$ & $0^{*}$ \\
\hline Chlorothalonil & 5 & 5 & 5 & 5 & 5 & 5 & 5 & 5 & 5 & 5 & 4 & 5 & 4 & 4 \\
\hline Manèbe & 4 & 4 & 3 & 3 & 2 & 2 & 1 & 1 & 1 & 1 & 1 & 1 & 0 & $0^{*}$ \\
\hline
\end{tabular}


manèbe, est actif, mais à la concentration de $3000 \mathrm{mg} / \mathrm{l}$, assurant une activité puissante et durable, la phytotoxicité est très élevée. Ainsi, les pulvérisations d'une solution de manèbe à $500 \mathrm{mg} / 1$ pratiquées sur les périmètres maraîchers du pays expliquent les échecs successifs des traitements et l'extension de la maladie. Par contre, ce produit, ou d'autres dithiocarbamates comme le mancozèbe, peu actifs sur $S$. floridanum, sont de bons moyens de lutte contre $S$. vesicarium de l'asperge (BLANCARD et al., 1984), S. botryosum de la tomate (ROTEM, 1968), de la laitue (SIVAN \& BARKAIGOLAN, 1976) ou de l'asperge (GINDRAT et al., 1984). Quant au zinèbe, il est actif sur $S$. sarciniforme de la vigne (CASSARINI \& PUCCI, 1956 ; CIFERI, 1961).

La faible action in vivo du manèbe, du chlorothalonil et du captafol pourrait être liée aux conditions tropicales. En effet, l'humidité est bien connue pour favoriser la décomposition du manèbe, la lumière celle du chlorothalonil (SIVAN \& BARKAI-GOLAN, 1976).
Avant d'envisager une lutte chimique contre un agent pathogène, il est donc indispensable d'étudier, in vitro, l'activité de différents fongicides puis d'expérimenter dans les conditions naturelles de vie de l'hôte et de son parasite. La sélection des produits les plus efficaces est alors rapide et leur comportement, in vivo, vérifié. Ainsi, l'emploi d'un fongicide ayant déjà fait la preuve de son activité sur des espèces voisines de champignons phytopathogènes sera préconisé ou proscrit.

Pour la stemphyliose de $S$. gilo, des applications bimensuelles de thirame à raison de $100 \mathrm{mg} / \mathrm{l}$ sont préconisées. Ce traitement, économique, performant et durable, doit accompagner des pratiques culturales défavorables à l'installation et à la propagation du parasite (CLERIVET, 1982).

Reçu le 28 juillet 1986. Accepté le II décembre 1986.

\section{RÉFÉRENCES BIBLIOGRAPHIQUES}

Blancard D., Piquemal J. P., Gindrat D., 1984. La Stemphyliose de l'Asperge. P.H.M., Rev. Hortic., 248, 27-30.

Cassarini B., Pucci E., 1956. Specificità d'azione degli anticrittogamici. Accad. Lincei, 21, 305-308.

Ciferi R., 1961. Sinergisma tra zineb y TMTD (Thiram). Not. Mal. Painte, 56, 3-19.

Clerivet A., 1982. Une maladie foliaire de Solanum gilo Raddli due à Stemphylium floridanum, Hannon et Weber, en Côte-d'Ivoire. Agronomie, 2 (10), 1015-1018.

Clerivet A., 1985. Etude de l'influence de quelques facteurs externes sur la croissance et la conidiogenèse de Stemphylium floridanum, Hannon et Weber. Bull. Soc. Myc. Fr., 101 (3), 237-248.
Gindrat D., Varady C., Neury G., 1984. Asperge : une nouvelle maladie du feuillage provoquée par un Stemphylium. Rev. suisse Vitic. Arboric. Hortic., 16 (2), 81-85.

Leroux P., 1984. La résistance des champignons phytopathogènes aux fongicides : conséquence de l'intensification de la lutte chimique ? Défense des végétaux, 227, 135-145.

Rotem J., 1968. Field trials for the control of Stemphylium botryosum f. lycopersici in tomatoes. Israel J. Agric. Res., 18 (2), 87-88.

Sivan I., Barkai-Golan R., 1976. In vitro and in vivo evaluation of fungicides for control of Stemphylium botryosum f. lactucae. In lettuce leaves. Phytoparasitica, 4 (1), 33-39. 